\title{
Occurrence, Persistence, and Virulence Potential of Listeria ivanovii in Foods and Food Processing Environments in the Republic of Ireland
}

\author{
Avelino Alvarez-Ordóñez, ${ }^{1}$ Dara Leong, ${ }^{1}$ Ciara A. Morgan, ${ }^{2}$ \\ Colin Hill, ${ }^{2}$ Cormac G. M. Gahan, ${ }^{2}$ and Kieran Jordan ${ }^{1}$ \\ ${ }^{1}$ Teagasc Food Research Centre, Moorepark, Fermoy, County Cork, Ireland \\ ${ }^{2}$ School of Microbiology, University College Cork, Cork, Ireland \\ Correspondence should be addressed to Avelino Alvarez-Ordóñez; avelino.alvarez-ordonez@teagasc.ie
}

Received 5 December 2014; Accepted 19 February 2015

Academic Editor: Philippe Lanotte

Copyright (c) 2015 Avelino Alvarez-Ordóñez et al. This is an open access article distributed under the Creative Commons Attribution License, which permits unrestricted use, distribution, and reproduction in any medium, provided the original work is properly cited.

\begin{abstract}
The aim of this study was to assess the occurrence of L. ivanovii in foods and food processing environments in Ireland, to track persistence, and to characterize the disease causing potential of the isolated strains. A total of 2,006 samples (432 food samples and 1,574 environmental swabs) were collected between March 2013 and March 2014 from 48 food business operators (FBOs) belonging to different production sectors (dairy, fish, meat, and fresh-cut vegetable). Six of the forty-eight FBOs had samples positive for L. ivanovii on at least one sampling occasion. L. ivanovii was present in fifteen samples (fourteen environmental samples and one food sample). All but one of those positive samples derived from the dairy sector, where L. ivanovii prevalence was $1.7 \%$. Six distinguishable pulsotypes were obtained by PFGE analysis, with one pulsotype being persistent in the environment of a dairy food business. Sequence analysis of the $\operatorname{sig} B$ gene showed that fourteen isolates belonged to L. ivanovii subsp. londoniensis, while only one isolate was L. ivanovii subsp. ivanovii. Cell invasion assays demonstrated that the majority of L. ivanovii strains were comparable to L. monocytogenes EGDe in their ability to invade CACO-2 epithelial cells whilst four isolates had significantly higher invasion efficiencies.
\end{abstract}

\section{Introduction}

The genus Listeria is at present comprised of fifteen low $\mathrm{G}+\mathrm{C}$ content Gram-positive species. These are the Listeria sensu stricto species $L$. monocytogenes, $L$. marthii, $L$. innocua, $L$. welshimeri, L. seeligeri, and L. ivanovii, the distantly related species $L$. grayi, and the very recently described species $L$. rocourtiae, L. fleischmannii, L. weihenstephanensis, L. floridensis sp. nov., $L$. aquatica sp. nov., $L$. cornellensis sp. nov., $L$. riparia sp. nov., and L. grandensis sp. nov. [1,2]. Of these, only $L$. monocytogenes and L. ivanovii are recognized as pathogenic for warm-blooded hosts. While L. monocytogenes causes a severe foodborne disease in humans as well as invasive infections in a range of other mammals, L. ivanovii is almost exclusively linked to infections in sheep and cattle, although sporadic cases of $L$. ivanovii associated human infections have been reported $[3,4]$.
Due to its foodborne transmission, research on L. monocytogenes has received special attention in the last decades. Indeed, studies on occurrence and distribution of L. monocytogenes in foods and food processing environments are numerous and report variable prevalence. As an example, recent surveys carried out in the United Kingdom [5], Greece [6], Sweden [7], Ireland [8, 9], and various countries in Europe (Austria, Romania, Spain, and the Slovak Republic) [10] have reported L. monocytogenes prevalence ranging from 2.5 to $38 \%$. There is less information available in the literature on the occurrence and distribution of other Listeria species along the food chain, although it appears that, apart from $L$. monocytogenes, $L$. innocua is the most frequently isolated Listeria species [11, 12]. Regarding L. ivanovii, a few reports exist which describe a low occurrence, generally of $<2 \%$ [11-13], although little or no information is available on its occurrence in Irish food industries. 
Bacterial persistence, defined as repeated isolation of an indistinguishable (by pulsed field gel electrophoresis [PFGE]) isolate at sampling times greater than 6 months, is a great concern for food industries since it can lead to the repeated contamination of food with spoilage or pathogenic microorganisms and has been demonstrated to recurrently happen for strains of L. monocytogenes [14]. A similar phenomenon could also occur for other members of the genus Listeria, including L. ivanovii. In fact, a study by Vázquez-Villanueva et al. [15] has provided evidence for the persistence of a $L$. ivanovii subsp. ivanovii isolate in a Spanish cheese factory. These authors found a common PFGE pulsotype in both ewe's and goat's raw milk batches tested over a 6-month period and on the inner surfaces of raw milk bulk tanks and the milk dump tank at the cheese factory.

Both L. monocytogenes and L. ivanovii are facultative intracellular bacteria capable of crossing the intestinal barrier and proliferating within macrophages and epithelial and endothelial cells and ultimately inducing cell-to-cell spread [16]. Interestingly, it is well known that L. monocytogenes isolates vary considerably with respect to virulence capacity and disease causing potential, with some isolates being incapable of invading gastrointestinal cells due to the expression of a truncated virulence factor, internalin A $[17,18]$. Whether similar heterogeneity in disease causing potential is also present in L. ivanovii remains unexplored.

The aim of this study was to assess the occurrence of $L$. ivanovii in foods and food processing environments in the Republic of Ireland, to track persistence of the isolates, and to characterize the disease causing potential of the isolated strains.

\section{Materials and Methods}

2.1. Detection of L. ivanovii in Food and Environmental Samples. From March 2013 to March 2014, a total of 48 food processing facilities from various food sectors, that is, dairy (18 facilities), meat (12 facilities), seafood (8 facilities), freshcut vegetable (6 facilities), and miscellaneous (4 facilities), were sampled bimonthly. The selection of food processing facilities allowed coverage of major geographic areas of the Republic of Ireland.

Sampling packs, which consisted of a polystyrene box (DS Smith, UK) containing six premoistened $3 \mathrm{M}$ sponge-stick swabs (Technopath, Ireland), a sterile liquid container (VWR, Ireland), two sterile bags (VWR, Ireland), two cable ties, and two ice packs, were sent to all participating food processing facilities. Food business operators (FBOs) received detailed instructions which included information on how to take swab samples, which areas to sample, the type of food samples required, and the packaging and shipment of the samples to the laboratory. For food samples, FBOs were instructed to send two food samples which were at the stage of being ready to be sent from the processing facility.

Every second month, FBOs took 6 environmental samples and sent them to the laboratory by overnight courier along with 2 food samples. Thirty-seven FBOs were initially enrolled in the monitoring programme and 11 further FBOs later showed their interest in joining the collaborative network at different stages during the sampling year. On the other hand, 3 FBOs no longer wished to take part in the analysis or went out of business and several other companies missed one or various sample submissions throughout the sampling period.

Samples were analyzed by following the ISO 11290-1 method for detection of L. monocytogenes, except that only one chromogenic agar was used. After the environmental swabs arrived at the laboratory, $100 \mathrm{~mL}$ of half Fraser broth (VWR, Ireland) was added to bags containing $3 \mathrm{M}$ sticksponge swabs, after which they were incubated at $30^{\circ} \mathrm{C}$ for $24 \mathrm{~h}$. Then, a $0.1 \mathrm{~mL}$ aliquot was transferred to $10 \mathrm{~mL}$ of full Fraser broth, which was further incubated at $37^{\circ} \mathrm{C}$ for $48 \mathrm{~h}$. In addition, a $0.02 \mathrm{~mL}$ aliquot of the 1st enrichment broth was plated onto Agar Listeria according to Ottaviani and Agosti (ALOA) agar plates (Biomérieux, UK), which were incubated at $37^{\circ} \mathrm{C}$ for $48 \mathrm{~h}$. After incubation of the full Frazer broth, $10 \mu \mathrm{L}$ was streaked onto ALOA agar plates, which were again incubated at $37^{\circ} \mathrm{C}$ for $48 \mathrm{~h}$. For liquid or food samples, $225 \mathrm{~mL}$ of half Fraser broth was added to $25 \mathrm{~mL}$ or $25 \mathrm{~g}$ of randomly selected analytical units of the food samples. Samples were then homogenized in a stomacher (Colworth Stomacher 400) for $4 \mathrm{~min}$ and incubated at $30^{\circ} \mathrm{C}$ for $24 \mathrm{~h}$. Subsequently, analysis of samples was continued by following the same approach used for environmental samples. After incubation, ALOA agar plates were examined for typical $L$. monocytogenes/L. ivanovii colonies (blue-green colonies with opaque halo).

After confirmation of L. monocytogenes/L. ivanovii isolates (performed as described below) sampling results were regularly communicated to collaborating FBOs.

2.2. Molecular Characterization of L. ivanovii Isolates. Two characteristic L. monocytogenes/L. ivanovii colonies for each positive enrichment were streaked first onto Brilliance Listeria Agar (BLA) plates (Fannin, Ireland), which were incubated at $37^{\circ} \mathrm{C}$ for $48 \mathrm{~h}$, and then onto Brain Heart Infusion (BHI) agar plates, which were incubated at $37^{\circ} \mathrm{C}$ for $24 \mathrm{~h}$. Cryoinstant tubes (VWR, Ireland) were prepared by resuspending the bacterial mass from $\mathrm{BHI}$ agar plates and were kept at $-20^{\circ} \mathrm{C}$ for bioconservation.

Isolates were differentiated as L. monocytogenes or $L$. ivanovii by multiplex PCR as described by Ryu et al. [19] and L. ivanovii were confirmed by $\operatorname{sig} B$ sequencing as described below. PFGE analyses with the restriction enzymes AscI and ApaI were carried out on all confirmed $L$. ivanovii isolates according to the International Standard PulseNet protocol [20]. Isolate similarity dendrograms were generated for PFGE analysis using the BioNumerics version 5.10 software (Applied Maths, Belgium), by the unweighted pair group method with arithmetic mean (UPGMA) with tolerance and optimization settings of $1 \%$, as previously described [21]. In addition, representative isolates from each pulsotype were subjected to real-time PCR analyses for differentiation of $L$. monocytogenes and L. ivanovii through amplification of $h l y$ as described by Rodríguez-Lázaro et al. [22] and of act $A$ as described by Oravcová et al. [23]. The sigB gene of L. ivanovii isolates was amplified using Taq DNA polymerase 
(Thermo Scientific, Ireland) with primers sigB-F (AATATATTAATGAAAAGCAGGTGGAG) and sigB-R (ATAAATTATTTGATTCAACTGCCTT) at $95^{\circ} \mathrm{C}$ for $2 \mathrm{~min}$, followed by 30 cycles of $95^{\circ} \mathrm{C}$ for $1 \mathrm{~min}, 55^{\circ} \mathrm{C}$ for $30 \mathrm{~s}, 72^{\circ} \mathrm{C}$ for $1 \mathrm{~min}$, and a final extension at $72^{\circ} \mathrm{C}$ for $5 \mathrm{~min}$. PCR products were purified with the QIAquick PCR Purification Kit (Qiagen, Ireland) and sequenced by Source Bioscience services. Phylogenetic relationships between sequences were analysed using the web service http://www.phylogeny.fr/ as described by Dereeper et al. [24].

2.3. Invasion of CACO-2 Cells by L. ivanovii Isolates. The epithelial cell invasion assay was based upon the protocol of Nightingale et al. [25]. CACO-2 human intestinal cells (originally derived from human colon adenocarcinoma) were routinely maintained and grown in Dulbecco Modified Eagle Medium (DMEM) (Sigma-Aldrich, Ireland), supplemented with 10\% Foetal Bovine Serum (Gibco, Ireland), 1\% Penicillin-Streptomycin (Sigma-Aldrich), and 1\% nonessential amino acids (Sigma-Aldrich) in a $37^{\circ} \mathrm{C}$ incubator supplemented with $5 \% \mathrm{CO}_{2}$. Cells were counted using a haemocytometer and trypan blue exclusion to a cell density of $2 \times 10^{5}$ cells/mL of medium and seeded into each well of a 24 -well tissue culture plate (Starstedt), in triplicate. Cells were allowed to grow to a confluency of $80 \%$ over $48 \mathrm{~h}$. Twenty-four hours prior to the assay, cells were washed and incubated in antibiotic-free DMEM.

Cultures of L. monocytogenes EGDe, L. monocytogenes $\mathrm{PMSC1}$, or L. ivanovii strains were grown overnight in $\mathrm{BHI}$ at $37^{\circ} \mathrm{C}$ with shaking. One $\mathrm{mL}$ of the overnight culture was subsequently pelleted by centrifugation and then washed in PBS, diluted to a final concentration of $2 \times 10^{7} \mathrm{CFU} / \mathrm{mL}$, and resuspended in antibiotic-free DMEM. Precise numbers of bacterial CFUs added to wells at $T_{0}$ were calculated subsequently following plate counts.

Growth medium was removed from the CACO-2 cells in each well and cells were washed once with sterile PBS and $1 \mathrm{~mL}$ of bacteria in antibiotic-free DMEM was added (giving a multiplicity of infection of 100). Cells were incubated for $1 \mathrm{~h}$ at $37^{\circ} \mathrm{C} / 5 \% \mathrm{CO}_{2}$ to allow for internalisation of the bacteria. Subsequently, the bacterial inoculum was removed and the monolayer was washed once with sterile PBS. Fifty $\mu \mathrm{g} / \mathrm{mL}$ gentamicin (Sigma) was resuspended in antibiotic-free DMEM, applied to the monolayer, and incubated for one further hour to kill extracellular bacteria. This was followed by lysis of the entire monolayer with ice cold sterile water containing $0.1 \%$ of TritonX-100. One hundred $\mu \mathrm{L}$ of the lysate was serially diluted and plated onto BHI agar (in triplicate for each well) which was incubated at $37^{\circ} \mathrm{C}$ overnight.

Data were expressed as mean \pm SEM of at least three biological replicate samples. Data were transformed to $\log$ base ten prior to one-way Analysis of Variance (ANOVA) which was used to test the significance of differences in three or more groups followed by a post hoc test (in this case, Dunnett). In all cases, $P<0.05$ was considered to be statistically significant. Graphs and statistical calculations were prepared using GraphPad Prism 5 (San Diego, California).

\section{Results}

From March 2013 to March 2014 a total of 2,006 samples (1,574 environmental samples and 432 food samples) were analyzed following the ISO 11290-1 standard methodology. L. ivanovii was present in fifteen of the 2,006 samples tested (prevalence of $0.75 \%$ ), accounting for 14 environmental samples and one food sample. All but one positive environmental sample derived from processing facilities of the dairy sector, where L. ivanovii prevalence was $1.7 \%$. These isolates were obtained from nonfood contact surfaces such as drains, floors, and pooled water on floors. The nondairy isolate was obtained from a seafood processing environment (floor), while the positive food sample was obtained from meat sausages. No positive samples were observed in processing facilities of the fresh-cut vegetable sector. It is important to note that only six of the forty-eight processing facilities analyzed had samples positive for L. ivanovii on at least one sampling occasion, with prevalence rates at those six facilities ranging from $1.8 \%$ to $13.1 \%$ (Table 1 ).

PFGE analysis was performed for all confirmed $L$. ivanovii isolates in order to track persistence events in the food processing environment (Figure 1). Six distinguishable pulsotypes were observed. In two dairy processing facilities (FBO 1 and FBO 12), L. ivanovii strains with indistinguishable PFGE profiles were isolated at various sampling times during the monitoring programme. For FBO 1, L. ivanovii isolates belonging to the same pulsotype were obtained from drains, floors, and pooled water on floors in May 2013, September 2013, November 2013, January 2014, and March 2014 (10month persistence). For FBO 12, two L. ivanovii strains with indistinguishable PFGE profiles were isolated from drains in March 2013 and July 2013.

In order to characterize the L. ivanovii isolates at the subsp. level, the $\operatorname{sig} B$ gene was sequenced for representatives of the six distinguishable pulsotypes (Figure 2). Analysis of $\operatorname{sig} B$ sequences showed that five of the six pulsotypes (which correspond to 14 of the 15 positive samples) belonged to $L$. ivanovii subsp. londoniensis, while the remaining pulsotype (T6, with only one strain isolated from meat sausages) was $L$. ivanovii subsp. ivanovii.

When incorporating the PFGE profiles obtained in the current study to the Listeria spp. collection of profiles available at Teagasc Food Research Centre Moorepark, it became apparent that several isolates originally confirmed as L. monocytogenes by following the real-time PCR approach described by Rodríguez-Lázaro et al. [22] presented PFGE profiles indistinguishable from the ones obtained in this study. Some of these strains were analyzed by multiplex PCR and actually confirmed as L. ivanovii (data not shown). Subsequently, the real-time PCR protocol described by Rodríguez-Lázaro and coauthors was applied to representative strains of the six pulsotypes observed in the present study (Figure 3(a)). Amplification of the target $h l y$ gene occurred for both $L$. monocytogenes positive control strains used, with Ct values of 17.9 and 18.2, while late amplification of the target gene was observed for the L. ivanovii isolates tested, with Ct values ranging from 26.1 to 32.7. In addition, the real-time PCR meth odology described by Oravcová et al. [23] for confirmation 


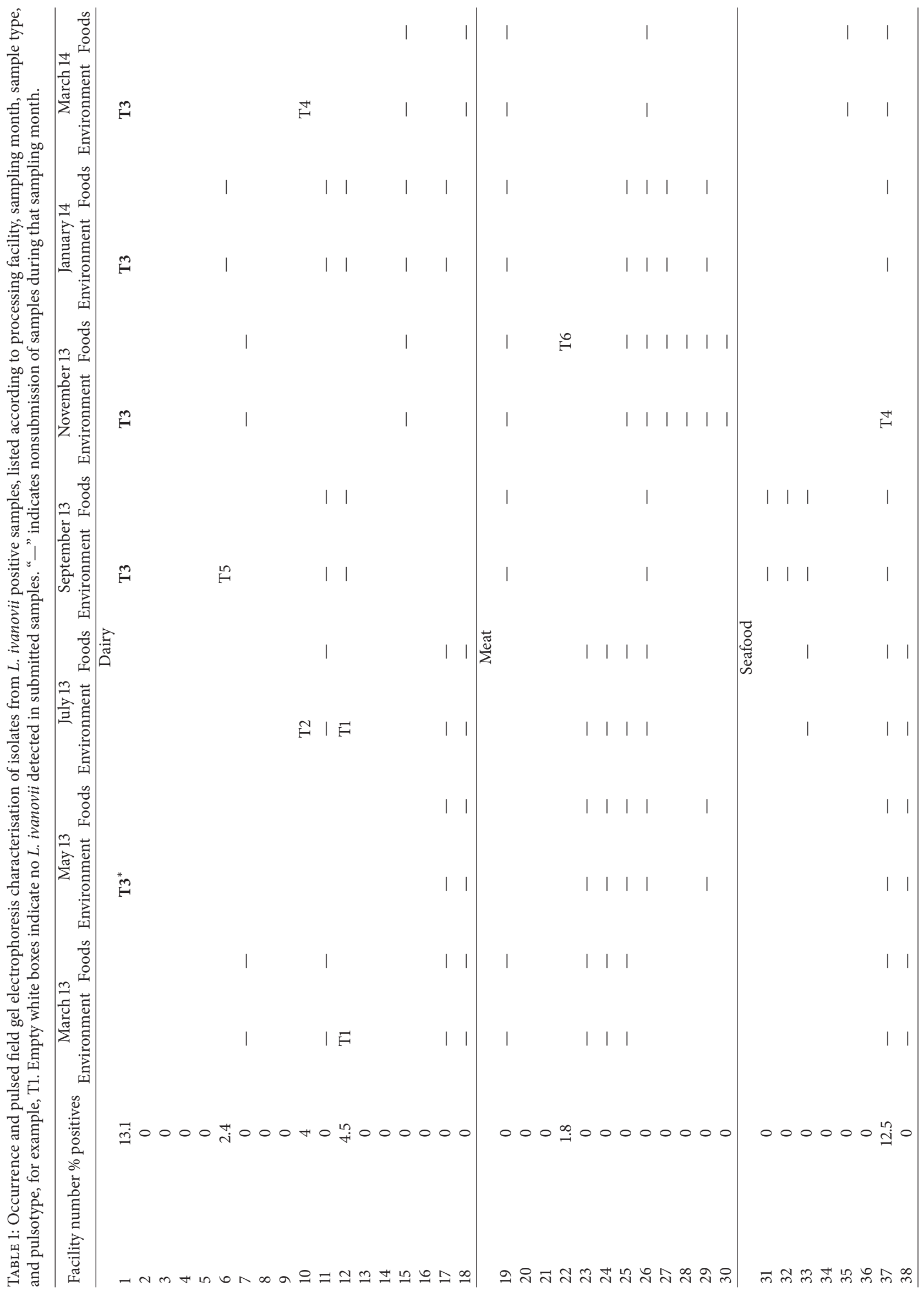




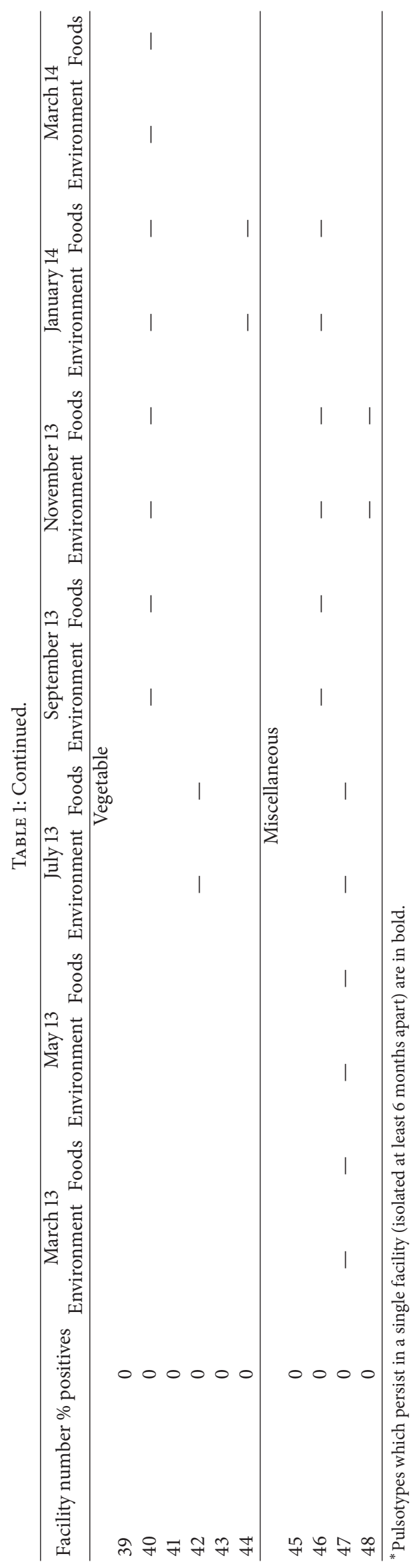



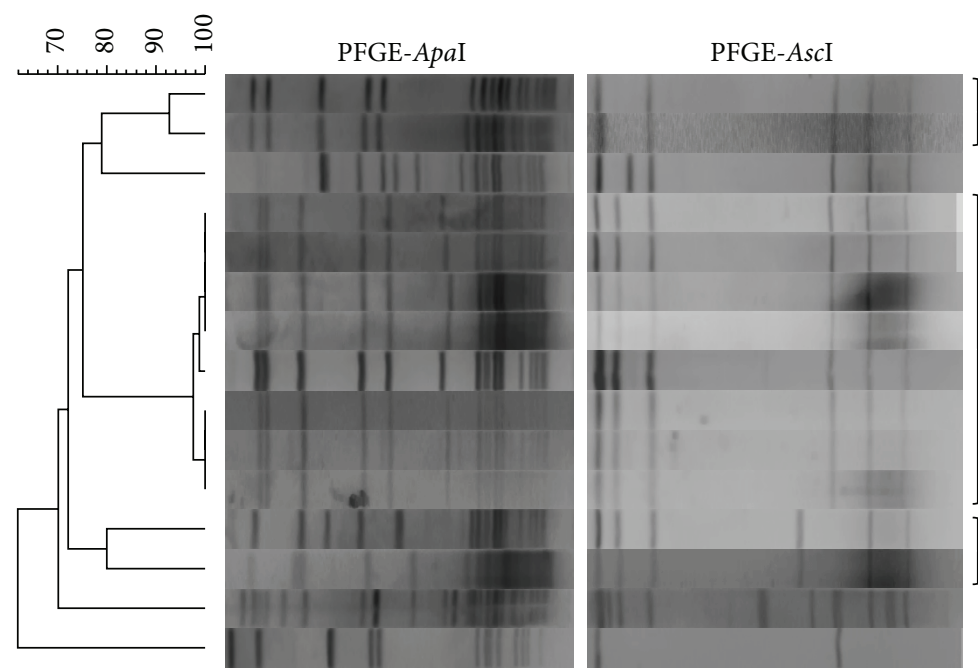

\begin{aligned} & \multicolumn{1}{|c}{$\begin{array}{l}\text { Location } \\ \text { T1 }\end{array}$} $\begin{array}{l}\text { Drain } \\ \text { Drain }\end{array} \\ &$ T2 2 Not stated \\ & Floor \\ & Pooled water \\ & Pooled water \\ & T3 Drain \\ & Drain \\ & Drain \\ & Pooled water \\ & Pooled water \\ & T4 Floor \\ & Not stated \\ & T5 Drain \\ & T6 Sausages \\ &\end{aligned}

$\begin{array}{ll}\text { Facility } & \text { Date isolated } \\ \text { FBO 12 } & \text { July 2013 } \\ \text { FBO 12 } & \text { March 2013 } \\ \text { FBO 10 } & \text { July 2013 } \\ \text { FBO 1 } & \text { September 2013 } \\ \text { FBO 1 } & \text { September 2013 } \\ \text { FBO 1 } & \text { November 2013 } \\ \text { FBO 1 } & \text { November 2013 } \\ \text { FBO 1 } & \text { January 2014 } \\ \text { FBO 1 } & \text { March 2014 } \\ \text { FBO 1 } & \text { March 2014 } \\ \text { FBO 1 } & \text { May 2013 } \\ \text { FBO 37 } & \text { November 2013 } \\ \text { FBO 10 } & \text { March 2014 } \\ \text { FBO 6 } & \text { September 2013 } \\ \text { FBO 22 } & \text { November 2013 }\end{array}$

FIGURE 1: Dendrogram of PFGE pulsotypes of Listeria ivanovii isolates obtained from food and processing environment samples from the Republic of Ireland analyzed from March 2013 to March 2014.

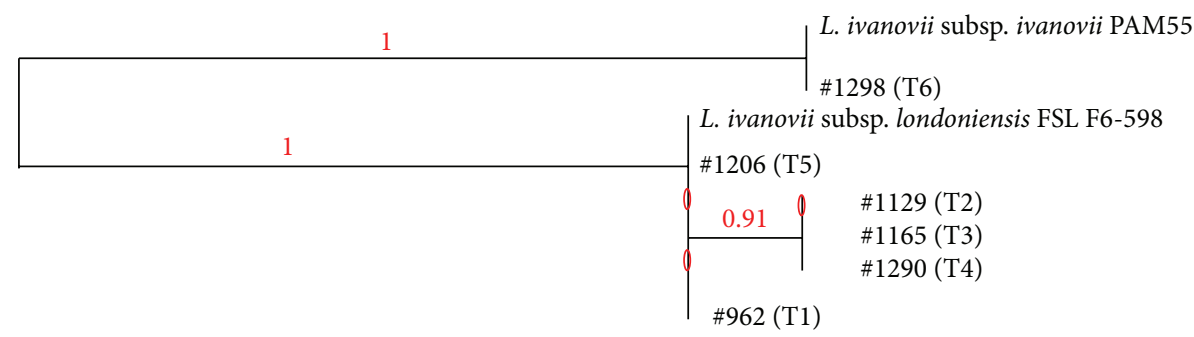

0.01

Figure 2: Phylogenetic tree (based on the sequence of the sigB gene) for the reference $L$. ivanovii subsp. ivanovii and $L$. ivanovii subsp. londoniensis strains and representatives of the six L. ivanovii pulsotypes found in the current study.

of L. monocytogenes based on the amplification of the actA gene was also tested with representative strains of the six $L$. ivanovii pulsotypes, and similarly late amplification events occurred, with $\mathrm{Ct}$ values ranging from 26.8 to 35.32 , in contrast to $\mathrm{Ct}$ values of 18.4 and 20.0 observed for $L$. monocytogenes isolates tested (Figure 3(b)).

In order to determine the ability of various $L$. ivanovii strains to invade gastrointestinal epithelial cells, a standardized CACO-2 invasion assay [25] was carried out. Representative strains from 4 of the 6 pulsotypes were compared to an invasive laboratory strain of L. monocytogenes (strain EGDe) as well as a noninvasive $L$. monocytogenes strain carrying a defined premature stop codon in the inlA gene (PMSC1) [25]. The assay clearly differentiates between invasive and noninvasive L. monocytogenes isolates (Figure 4) and invasion efficiency of wild-type L. monocytogenes and the PMSC1 strain were roughly equivalent to results in previous studies $[25,26]$. L. ivanovii strains were generally highly invasive with 7 out of 9 strains demonstrating levels of invasion that were equal to or higher than those of L. monocytogenes EGDe. Two strains (1261 and 1167) were moderately less invasive than L. monocytogenes EGDe, but none of the isolates demonstrated an invasion phenotype that was comparable to the PMSC1 $L$. monocytogenes isolate. Interestingly, four L. ivanovii isolates $(1017,1165,1262$, and 1290$)$ were significantly $(P<0.05)$ more invasive than L. monocytogenes EGDe.

\section{Discussion}

The occurrence of L. ivanovii in foods and food processing environments was evaluated for the first time in the Republic of Ireland by bimonthly testing, over a one-year period, samples from forty-eight processing facilities. The observed L. ivanovii prevalence was in general low $(0.75 \%)$. This agrees with the few reports available in the literature which also describe low $L$. ivanovii prevalence in the range $0-2 \%$ [11-13]. However, the results showed that L. ivanovii occurrence depended on the food sector. Thus, while a higher prevalence of $1.7 \%$ was observed for the dairy sector, very low prevalences $(0.2 \%$ and $0.3 \%$, resp.) were found for the meat and seafood sectors and no positive samples at all were obtained for the fresh-cut vegetable industry sector (278 samples analysed). It is important to note that $L$. ivanovii predominantly infects small ruminants and cattle, which can act as reservoirs. Ruminants can carry L. ivanovii and 


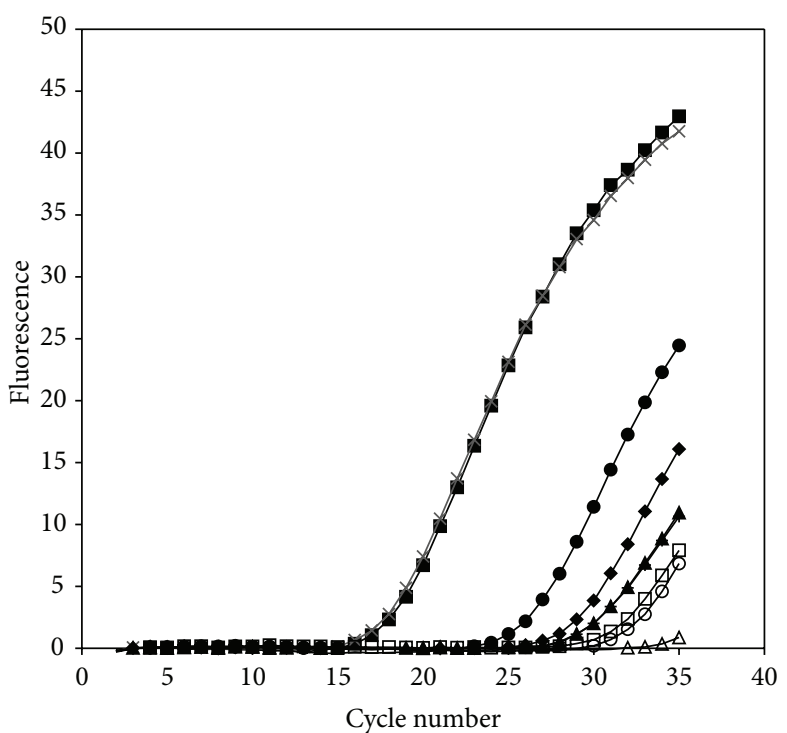

$\begin{array}{ll}\rightarrow-\text { L. monocytogenes } 1 / 2 \mathrm{a} & \square-\text { L. ivanovii \#1290 (T4) } \\ \rightarrow \text { L. monocytogenes } 4 \mathrm{~b} & - \text { L. ivanovii \#1206 (T5) } \\ \rightarrow \text { L. ivanovii \#962 (T1) } & - \text { L. ivanovii \#1298 (T6) } \\ \rightarrow \text { L. ivanovii \#1129 (T2) } & \triangle \text { Negative control } \\ - \text { - L. ivanovii \#1165 (T3) } & \end{array}$

(a)

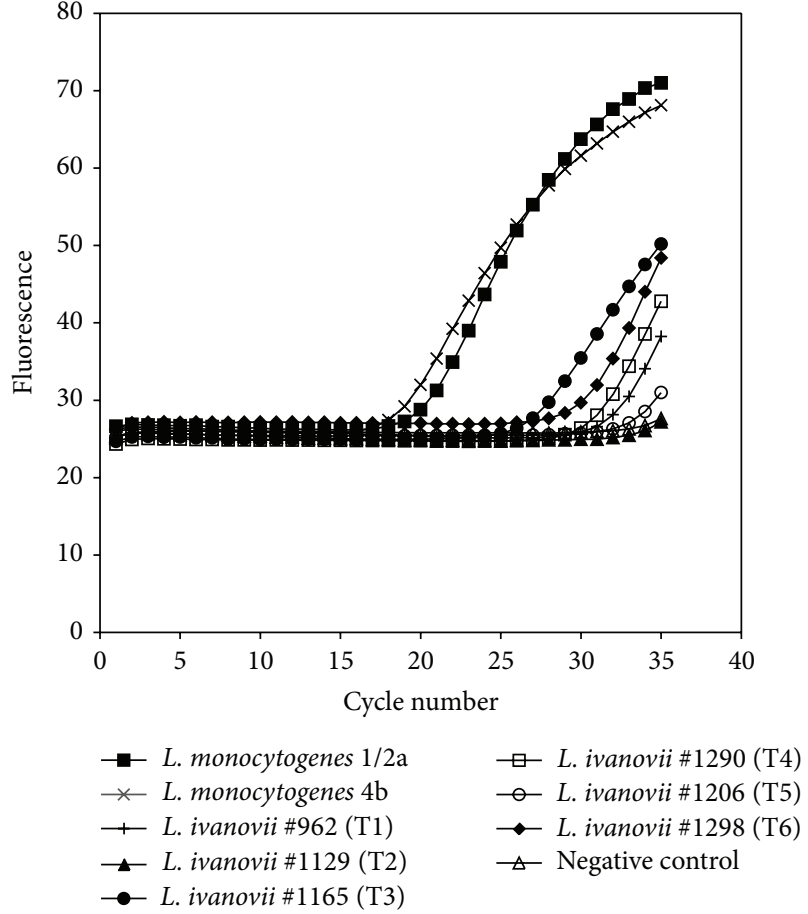

(b)

FIgURe 3: Amplification plot for $h l y$ (a) and actA (b) in L. ivanovii following the rt-PCR methodology described by Rodríguez-Lázaro et al. [22] and Oravcová et al. [23], respectively.

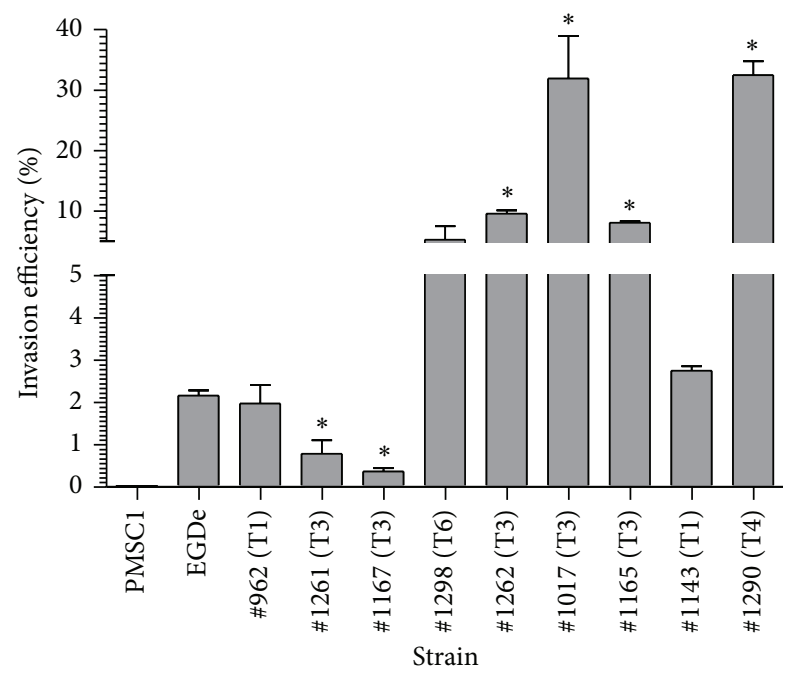

FIGURE 4: Invasive potential of wild-type L. ivanovii isolates in a CACO-2 epithelial cell assay. The strains were incubated with CACO-2 cells in vitro for one hour and levels of bacterial invasion were subsequently measured. For comparison, invasive (EGDe) and noninvasive (PMSC1) strains of L. monocytogenes were also examined. Data represents \% invasion efficacy (relative to Listeria numbers initially added per well). Statistical significance was determined using one-way ANOVA and the Dunnett post hoc test with all strains compared to L. monocytogenes EGDe $\left({ }^{*} P<0.05\right)$. All strains displayed statistically higher $(P<0.05)$ levels of invasion efficiency relative to the PMSC1 strain. contamination of milk can occur. Interestingly, three of the four dairy business operators that had positive samples (FBO 1, FBO 10, and FBO 12) produce cheese using milk from their own herds of cows or goats. Farming activity is carried out in those cases at facilities close to the cheese making facilities. This may potentially pose a further risk of processing environment contamination by L. ivanovii.

A survey regarding L. monocytogenes occurrence was conducted in parallel and showed that $L$. monocytogenes was present in $4.6 \%$ of samples analysed, with similar rates in food and environmental samples [9]. In most sampling occasions when L. ivanovii was detected no L. monocytogenes contamination was observed. However, there were three sampling occasions (Facility number 1: Environment, May 13; Facility number 1: Environment, November 13; Facility number 22: Foods, November 13) at which both L. ivanovii and L. monocytogenes isolates were identified, and in the particular case of Facility no. 1 both $L$. ivanovii and L. monocytogenes were isolated from the same samples (a drain and pooled water in the wash room) on November 13.

Molecular analysis of L. ivanovii isolates obtained throughout the monitoring programme showed that fourteen of the fifteen isolates (including all dairy isolates) belonged to L. ivanovii subsp. londoniensis, while only an isolate from meat sausages was L. ivanovii subsp. ivanovii. Interestingly, all environmental isolates were L. ivanovii subsp. londoniensis, while the only food isolate was L. ivanovii subsp. ivanovii. Whether L. ivanovii subsp. londoniensis is widely more 
prevalent in the environments than L. ivanovii subsp. ivanovii or this is a particular phenomenon observed in processing facilities in Ireland remains to be elucidated.

Persistence of L. ivanovii, considered for this study as the detection of isolates with indistinguishable PFGE profiles at times six months or more apart, was observed for a cheese processing facility (FBO 1), where a persistent L. ivanovii subsp. londoniensis pulsotype (T3) was detected repeatedly over a 10-month period (from May 2013 to March 2014) in several nonfood contact environments (drains, floors, and pooled water on floors). In addition, another pulsotype (T1), which cannot yet be considered as persistent, was found in drains of a cheese factory (FBO 12) at times four months apart (March to July 2013). These two cheese processing facilities were the ones with the highest $L$. ivanovii occurrence (13.1\% and $4.5 \%$, resp.). Long-term survival of strains in a food processing facility, such as these, confers a higher risk of bacterial transfer to food and therefore a higher risk of human exposure to the microorganism. Bacterial persistence in food processing environments can be due to the existence of harborage sites that are colonized by bacteria and cannot be effectively cleaned or disinfected or can be due to the enhanced ability of some particular strains to grow or survive and therefore persist in industrial settings [27]. Thus, strains with increased resistance to sanitizers, higher adaptability to stress, or better ability to form biofilms might be better suited to persist in inhospitable environments such as those prevailing in food industries. Persistence of $L$. ivanovii in food processing environments has been also previously reported by Vázquez-Villanueva et al. [15] who identified a persistent $L$. ivanovii subsp. ivanovii pulsotype from ewe's and goat's raw milk samples from asymptomatic animals at farm level and from swabs obtained from the inner surfaces of raw milk truck tanks and the milk dump tank at the cheese factory level.

The current study also gives evidences that misidentification of $L$. ivanovii isolates as $L$. monocytogenes could occur when following the standard methodology for detection of $L$. monocytogenes in food and environmental samples. $L$. ivanovii strains are phosphatidylinositol-specific phospholipase $\mathrm{C}$ positive, and as such they grow in standard selective L. monocytogenes chromogenic agar plates forming colonies with the same characteristics as L. monocytogenes (blue-green colonies surrounded by an opaque halo on ALOA plates). Genes within the prfA virulence gene cluster are habitually used as target genes for $L$. monocytogenes confirmation PCR methodologies (e.g., hly and actA). The prfA virulence gene cluster is present between the prs and ldh genes in the pathogenic L. monocytogenes and $L$. ivanovii but is absent from the nonpathogenic Listeria species [28]. Two widely used rt-PCR methodologies specifically designed for the detection and quantification of L. monocytogenes and based on the amplification of the $h l y$ and actA genes $[22,23]$ were applied to the set of $L$. ivanovii strains isolated in the present study. The results showed that a late amplification (but earlier than the negative control) of both target genes occurred for L. ivanovii isolates, which could lead to an erroneous interpretation of results. Indeed, the Teagasc Food Research Centre Moorepark culture collection contained various strains originally classified as L. monocytogenes by following the approach described by Rodríguez-Lázaro et al. [22] that were subsequently identified as $L$. ivanovii during the course of this study. These results show the need for finetuning of the currently available molecular methodologies for confirmation of L. monocytogenes. Incorporation of such molecular tools able to rapidly and successfully discriminate $L$. ivanovii from $L$. monocytogenes is also advisable when implementing monitoring programmes focused on L. monocytogenes.

L. ivanovii is known to cause disease predominately in ruminants but has been associated on occasions with human disease $[3,4]$ and is considered to be a potential opportunistic pathogen of humans [4]. To date, studies examining the virulence characteristics of $L$. ivanovii have examined individual reference strains rather than collections of isolates. These studies indicate that $L$. ivanovii is capable of cellular invasion, often at levels in excess of L. monocytogenes [29-31]. L. ivanovii is also capable of lysis of the host cell phagosome and actin polymerization but is perhaps less effective than L. monocytogenes in cell-to-cell spread and intracellular multiplication $[29,31,32]$. The findings of this study support previous studies and demonstrated that some wild-type isolates of $L$. ivanovii are more invasive than a clinical $L$. monocytogenes reference isolate (EGDe). Indeed, the majority of isolates in this study were capable of highly effective cellular invasion, suggestive of some degree of disease causing potential. Further analysis is needed to ascertain the precise disease risk associated with these strains but the results suggest that such isolates may pose a health risk for immunocompromised individuals [4].

In conclusion, $L$. ivanovii prevalence in foods and food processing environments in the Republic of Ireland is low but cannot be considered negligible in processing facilities from the dairy sector, where contamination of environments through contaminated raw milk and persistence of isolates with good abilities to grow/survive in industrial settings in particular environments can occur, leading to a higher risk of contamination of processed foods. Although L. ivanovii is mainly linked to infections in sheep and cattle, recent reports have highlighted its disease causing potential in humans $[3,4]$ and the findings of this study demonstrated that the strains described are capable of invasion of human epithelial cells in vitro. These findings emphasize the need for dairy processors to be vigilant in order to avoid potential public health risks associated to $L$. ivanovii contamination.

\section{Conflict of Interests}

The authors declare that there is no conflict of interests regarding the publication of this paper.

\section{Acknowledgments}

The authors wish to acknowledge the cooperation of all food business operators involved in this work. Additional thanks are due to the Veterinary Food Safety Laboratory, Cork County Council. This work was supported by the Irish 
Department of Agriculture and Food and the Marine under the Food Institutional Research Measure (FIRM) Project no. 11F008. A. Alvarez-Ordóñez is a Starting Investigator Research Fellow funded by Science Foundation Ireland (SFI) under Grant no. 13/SIRG/2157.

\section{References}

[1] H. C. den Bakker, C. A. Cummings, V. Ferreira et al., "Comparative genomics of the bacterial genus Listeria: genome evolution is characterized by limited gene acquisition and limited gene loss," BMC Genomics, vol. 11, no. 1, article 688, 2010.

[2] H. C. den Bakker, S. Warchocki, E. M. Wright et al., "Listeria floridensis sp. nov., Listeria aquatica sp. nov., Listeria cornellensis sp. nov., Listeria riparia sp. nov. and Listeria grandensis sp. nov., from agricultural and natural environments," International Journal of Systematic and Evolutionary Microbiology, vol. 64, part 6, pp. 1882-1889, 2014.

[3] Y. M. Snapir, E. Vaisbein, and F. Nassar, "Low virulence but potentially fatal outcome-Listeria ivanovii," European Journal of Internal Medicine, vol. 17, no. 4, pp. 286-287, 2006.

[4] C. Guillet, O. Join-Lambert, A. le Monnier et al., "Human listeriosis caused by Listeria ivanovii," Emerging Infectious Diseases, vol. 16, no. 1, pp. 136-138, 2010.

[5] R. J. Meldrum, P. W. Ellis, P. T. Mannion, D. Halstead, and J. Garside, "Prevalence of Listeria monocytogenes in ready-to-eat foods sampled from the point of sale in wales, United Kingdom," Journal of Food Protection, vol. 73, no. 8, pp. 1515-1518, 2010.

[6] I. Sakaridis, N. Soultos, E. Iossifidou, A. Papa, I. Ambrosiadis, and P. Koidis, "Prevalence and antimicrobial resistance of Listeria monocytogenes isolated in chicken slaughterhouses in Northern Greece," Journal of Food Protection, vol. 74, no. 6, pp. 1017-1021, 2011.

[7] S. T. Lambertz, C. Nilsson, A. Brådenmark et al., "Prevalence and level of Listeria monocytogenes in ready-to-eat foods in Sweden 2010," International Journal of Food Microbiology, vol. 160, no. 1, pp. 24-31, 2012.

[8] B. K. Khen, O. A. Lynch, J. Carroll, D. A. Mcdowell, and G. Duffy, "Occurrence, antibiotic resistance and molecular characterization of Listeria monocytogenes in the beef chain in the Republic of Ireland," Zoonoses and Public Health, 2014.

[9] D. Leong, A. Alvarez-Ordóñez, and K. Jordan, "Monitoring occurrence and persistence of Listeria monocytogenes in foods and food processing environments in the Republic of Ireland," Frontiers in Microbiology, vol. 5, article 436, 2014.

[10] M. Muhterem-Uyar, M. Dalmasso, A. S. Bolocan et al., "Environmental sampling for Listeria monocytogenes control in food processing facilities reveals three contamination scenarios," Food Control, vol. 51, pp. 94-107, 2015.

[11] L. Chambel, M. Sol, I. Fernandes et al., "Occurrence and persistence of Listeria spp. in the environment of ewe and cow's milk cheese dairies in Portugal unveiled by an integrated analysis of identification, typing and spatial-temporal mapping along production cycle," International Journal of Food Microbiology, vol. 116, no. 1, pp. 52-63, 2007.

[12] S. Gebretsadik, T. Kassa, H. Alemayehu, K. Huruy, and N. Kebede, "Isolation and characterization of Listeria monocytogenes and other Listeria species in foods of animal origin in Addis Ababa, Ethiopia," Journal of Infection and Public Health, vol. 4, no. 1, pp. 22-29, 2011.
[13] P. C. Antoniollo, S. B. Fda, M. M. Jantzen, E. H. Duval, and W. P. da Silva, "Prevalence of Listeria spp. in feces and carcasses at a lamb packing plant in Brazil," Journal of Food Protection, vol. 66, no. 2, pp. 328-330, 2003.

[14] E. Fox, K. Hunt, M. O’Brien, and K. Jordan, "Listeria monocytogenes in Irish Farmhouse cheese processing environments," International Journal of Food Microbiology, vol. 145, pp. S39S45, 2011.

[15] J. Vázquez-Villanueva, B. Orgaz, S. Ortiz, V. López, J. V. Martínez-Suárez, and C. Sanjose, "Predominance and persistence of a single clone of Listeria ivanovii in a manchego cheese factory over 6 months," Zoonoses and Public Health, vol. 57, no. 6, pp. 402-410, 2010.

[16] J. A. Vázquez-Boland, M. Kuhn, P. Berche et al., "Listeria pathogenesis and molecular virulence determinants," Clinical Microbiology Reviews, vol. 14, no. 3, pp. 584-640, 2001.

[17] C. Jacquet, M. Doumith, J. I. Gordon, P. M. V. Martin, P. Cossart, and M. Lecuit, "A molecular marker for evaluating the pathogenic potential of foodborne Listeria monocytogenes," Journal of Infectious Diseases, vol. 189, no. 11, pp. 2094-2100, 2004.

[18] A. van Stelten, J. M. Simpson, T. J. Ward, and K. K. Nightingale, "Revelation by single-nucleotide polymorphism genotyping that mutations leading to a premature stop codon in inlA are common among Listeria monocytogenes isolates from readyto-eat foods but not human listeriosis cases," Applied and Environmental Microbiology, vol. 76, no. 9, pp. 2783-2790, 2010.

[19] J. Ryu, S. H. Park, Y. S. Yeom et al., "Simultaneous detection of Listeria species isolated from meat processed foods using multiplex PCR," Food Control, vol. 32, no. 2, pp. 659-664, 2013.

[20] PulseNet USA, International Standard PulseNet Protocol, PulseNet USA, 2009, http://www.cdc.gov/pulsenet/pathogens/ listeria.html

[21] E. M. Fox, N. Delappe, P. Garvey et al., "PFGE analysis of Listeria monocytogenes isolates of clinical, animal, food and environmental origin from Ireland," Journal of Medical Microbiology, vol. 61, no. 4, pp. 540-547, 2012.

[22] D. Rodríguez-Lázaro, M. Hernández, M. Scortti, T. Esteve, J. A. Vázquez-Boland, and M. Pla, "Quantitative detection of Listeria monocytogenes and Listeria innocua by real-time PCR: assessment of hly, iap, and lin02483 targets and AmpliFluor technology," Applied and Environmental Microbiology, vol. 70, no. 3, pp. 1366-1377, 2004.

[23] K. Oravcová, E. Kaclíková, K. Krascsenicsová et al., "Detection and quantification of Listeria monocytogenes by $5^{\prime}$-nuclease polymerase chain reaction targeting the actA gene," Letters in Applied Microbiology, vol. 42, no. 1, pp. 15-18, 2006.

[24] A. Dereeper, V. Guignon, G. Blanc et al., "Phylogeny.fr: robust phylogenetic analysis for the non-specialist," Nucleic Ccids Research, vol. 36, pp. W465-W469, 2008.

[25] K. K. Nightingale, K. Windham, K. E. Martin, M. Yeung, and M. Wiedmann, "Select Listeria monocytogenes subtypes commonly found in foods carry distinct nonsense mutations in inlA, leading to expression of truncated and secreted internalin A, and are associated with a reduced invasion phenotype for human intestinal epithelial cells," Applied and Environmental Microbiology, vol. 71, no. 12, pp. 8764-8772, 2005.

[26] L. Ciolacu, A. I. Nicolau, M. Wagner, and K. Rychli, "Listeria monocytogenes isolated from food samples from a Romanian black market show distinct virulence profiles," International Journal of Food Microbiology, 2014. 
[27] B. Carpentier and O. Cerf, "Review-persistence of Listeria monocytogenes in food industry equipment and premises," International Journal of Food Microbiology, vol. 145, no. 1, pp. $1-8,2011$.

[28] S. Cai and M. Wiedmann, "Characterization of the prfA virulence gene cluster insertion site in non-hemolytic Listeria spp.: probing the evolution of the Listeria virulence gene island," Current Microbiology, vol. 43, no. 4, pp. 271-277, 2001.

[29] I. Karunasagar, G. Krohne, and W. Goebel, "Listeria ivanovii is capable of cell-to-cell spread involving actin polymerization," Infection and Immunity, vol. 61, no. 1, pp. 162-169, 1993.

[30] W. F. Schlech III, Q. Luo, G. Faulkner, and S. Galsworthy, "Interaction of Listeria species with human cell monolayers," Clinical and Investigative Medicine, vol. 17, no. 1, pp. 9-17, 1994.

[31] C. Longhi, M. G. Ammendolia, M. P. Conte, L. Seganti, F. Iosi, and F. Superti, "Listeria ivanovii ATCC 19119 strain behaviour is modulated by iron and acid stress," Food Microbiology, vol. 42, pp. 66-71, 2014.

[32] F. Engelbrecht, G. Dominguez-Bernal, J. Hess et al., "A novel PrfA-regulated chromosomal locus, which is specific for Listeria ivanovii, encodes two small, secreted internalins and contributes to virulence in mice," Molecular Microbiology, vol. 30, no. 2, pp. 405-417, 1998. 

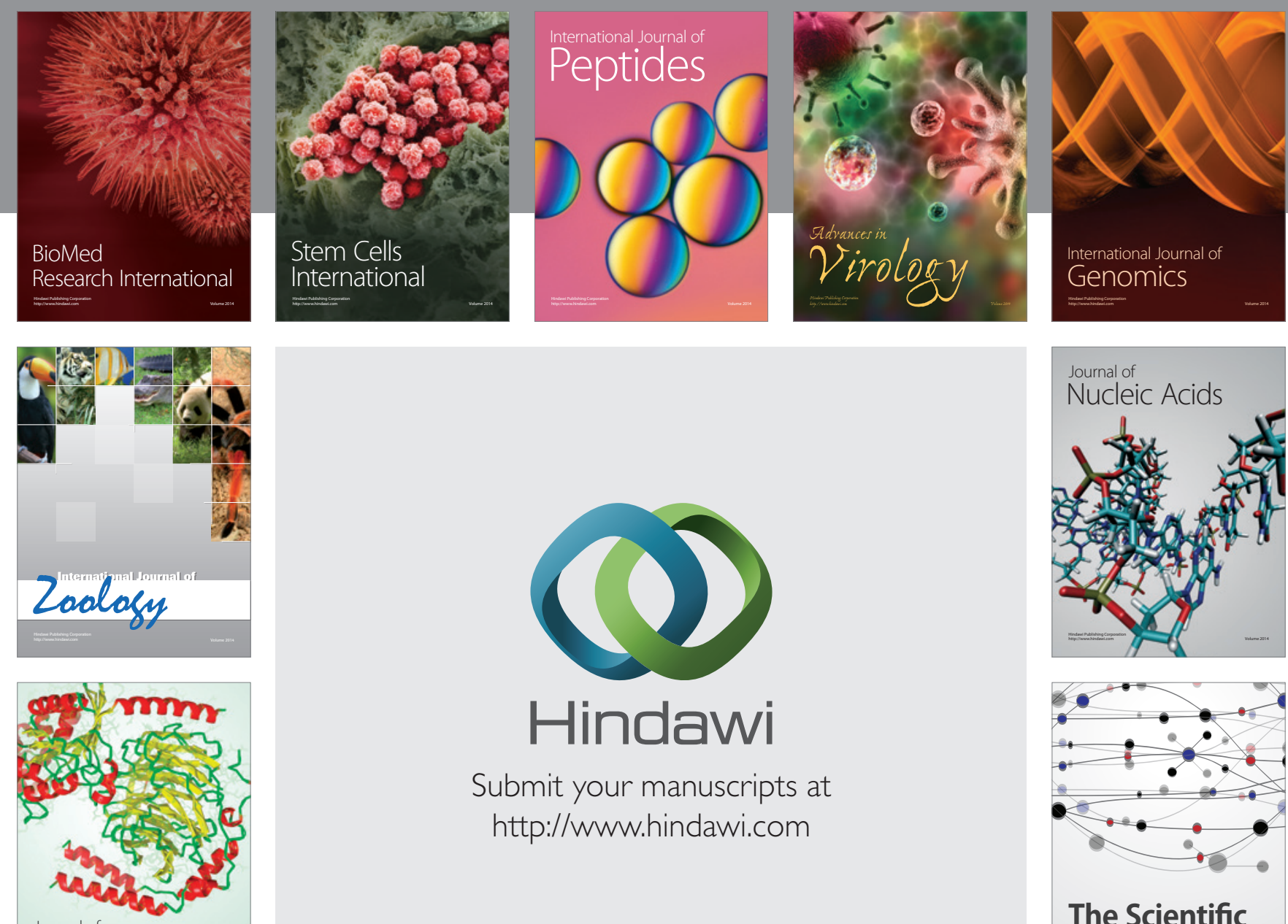

Submit your manuscripts at

http://www.hindawi.com

Journal of
Signal Transduction
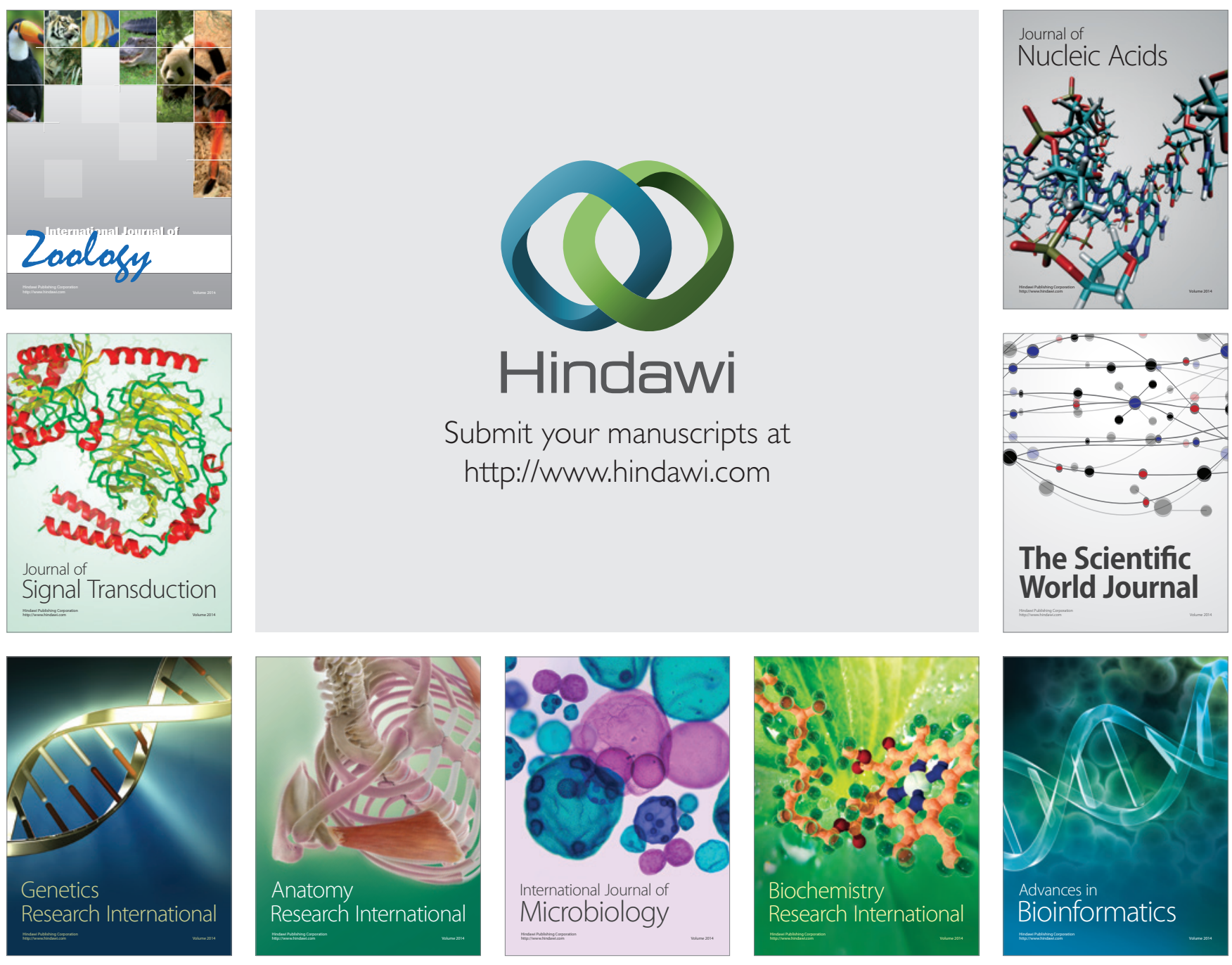

The Scientific World Journal
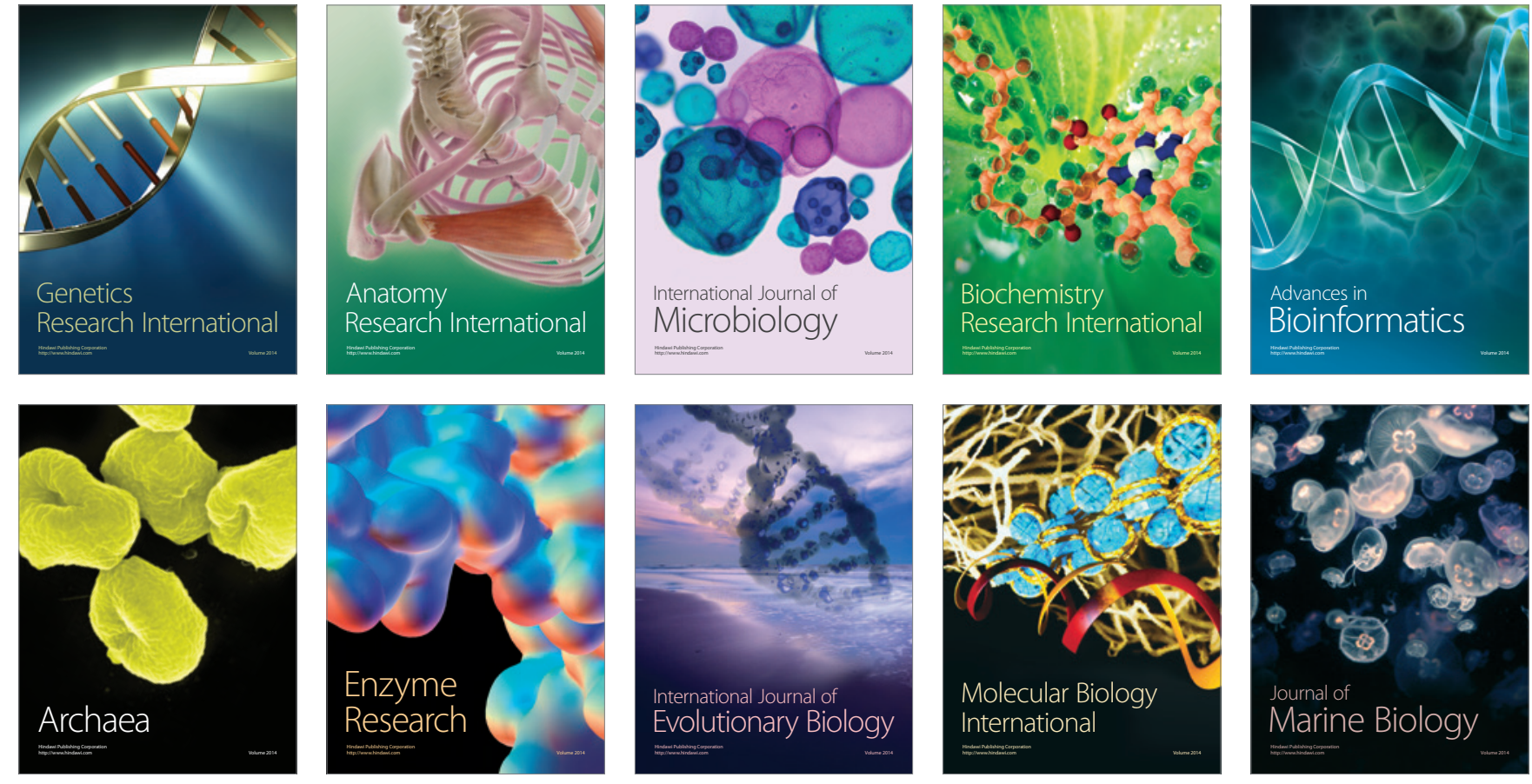